\title{
ROZPRAWY
}

ALBERT BOESTEN-STENGEL

\section{DANZIG AM MITTELMEER. DIE BRONZESKULPTUR DES NEPTUNBRUNNENS - IKONOGRAPHIE, BILDERFINDUNG UND BEDEUTUNG*}

Bei aller Ungewißheit, wer der unmittelbare Schöpfer ${ }^{1}$ (Abb. 1) des Danziger Bronze-Neptun gewesen sei, fehlt doch in keiner neueren Untersuchung der Name des in Florenz wirkenden Flamen Giambologna (Jean Boulogne, 1529-1608). Diese künstlerische Provenienz schien sich auch mit der Überlieferung zu vertragen, daß die Danziger in Augsburg um künstlerische und technische Unterstützung für ihr Projekt nachsuchten $^{2}$. Eben dort waren um 1600 gleich mehrere Schüler Giambolog-

* Editorische Anmerkung des Verfassers: Der vorliegende Text ist die Druckfassung des auf der Konferenz „Rzeźba w Prusach Królewskich. - Die Skulptur in KöniglichPreußen. III sesja naukowa z cyklu Sztuka i kultura w Prusach Królewskich" (Gdańsk, Muzeum Narodowe 18.-19.09.2009) gehaltenen Vortrags. Er hat, wie ich meine, seine Aktualität behalten. Erst nach meinem Vortrag erschien das Buch von Marcin Kaleciński, Mity Gdańska. Antyk w publicznej sztuce protestanckiej res publiki, Gdańsk 2011, auf dessen Kapitel 9.1 hier für eine zukünftige Diskussion hingewiesen sein soll.

${ }^{1}$ H. Carl, Der Neptunsbrunnen auf dem Langen Markte zu Danzig. Seine Entstehung und Geschichte, „Zeitschrift für Kunstgeschichte” 6, 1937, S. 163-165, ermittelte im Staatsarchiv Danzig aus den städtischen Rechnungsbüchern der Rechtsstadt, daß ein gewisser Peter Husen (Hussen) als „Bossierer” 1612-1615 den Guß vorbereitet, überwacht und nachbearbeitet haben sollte. Carl hielt ihn auch für den Urheber des Gußmodells. Bekannt ist von Peter Husen ferner die Bronzegruppe Löwe, der ein Pferd gerissen hat (nach dem Fragment einer Antike in Rom, Palazzo dei Conservatori), modelliert 1617 und gegossen 1624 für die Neugründung Glückstadt (dänisch: Lykstad) an der Unterelbe, seit 1647 im königlichen Garten in Kopenhagen. Daß Husen ein „Schüler” des aus Oberschwaben gebürtigen Hans Reichel gewesen sei, wie Carl aus dem Stilvergleich folgert, entbehrt bislang jeder Faktizität. Die Frage nach der Herkunft des Figurenmodells ist weiter offen. Zu Peter Husen in Kopenhagen vgl. L.O. Larsson, Bemerkungen zur Bildhauerkunst am dänischen Hofe im 16. und 17. Jahrhundert, (in:) Wege nach Süden, Wege nach Norden, Kiel 1998, S. 130-131.

2 Der im Original verschollene Brief des Augsburger Gießers Neidhart an den Danziger Stadtbaumeister Abraham van den Blocke, der nur in der Publikation durch Hirsch 1852 und dort mit dem Datum „30. Januar 1620” bekannt ist, bezeugt eine Reise des Abraham nach Augsburg, wo er einen geeigneten Bildhauer und Gießer gewinnen wollte. Neidhart empfahl demnach Adrian de Vries, nannte aber auch Hans Reichel. Für Krieg- 
nas tätig: Hubert Gerhard, Adriaen de Vries und Hans Reichel (Reichle). Giambologna erhielt seine wesentliche Formation und leistete seinen wichtigsten Beitrag in Florenz.

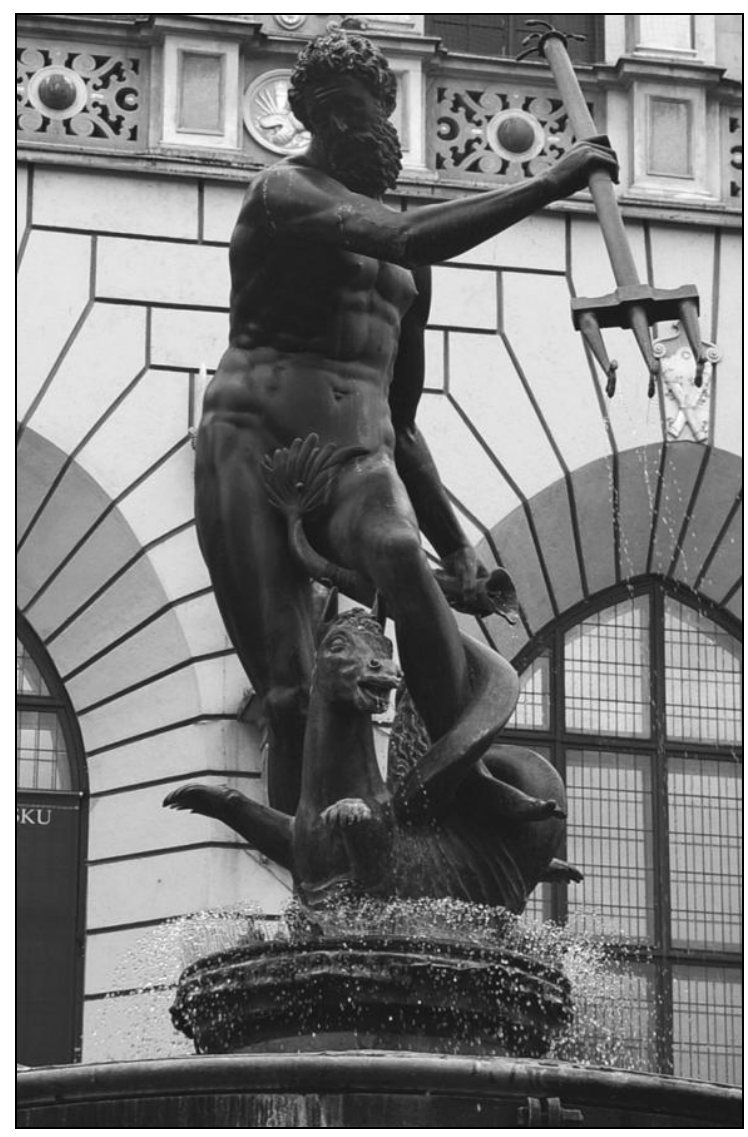

Abb. 1. Neptun, Danzig, Neptunbrunnen

baum war das ein weiteres Indiz eines Aufenthalts Reichels in Danzig um oder nach 1620. Carl bezweifelte, daß das Datum des Briefs richtig wiedergegeben sei, hielt den Vorgang selbst aber um 1610 für möglich, vgl. T. Hirsch, Zur Kunstgeschichte Danzigs. Der Springbrunnen auf dem Langen Markte, „Neue Preußische Provinzialblätter”, N.F., 2, 1852, S. 161; C. Buchwald, Adriaen de Vries, Leipzig 1899, S. 80-83; F. Kriegbaum, Hans Reichle, „Jahrbuch der Kunsthistorischen Sammlungen in Wien”, N.F., 5 (41), 1931, S. 201; H. Carl, op. cit., S. 153; T. Grzybkowska, Bildhauerei des 17. und 18. Jahrhunderts, (in:) Danziger Kunst vom 15. bis 18. Jahrhundert (Deutsche Ausgabe: Aurea Porta Rzeczypospolitej. Sztuka Gdańska od połowy XV do końca XVIII wieku. Eseje, katalog wystawy, Muzeum Narodowe w Gdańsku, red. T. Grzybkowska, Gdańsk 1997), red. T. Grzybkowska, Gdańsk 1997, S. 145, versteht die bisherige Chronologie der Dokumente dahin, daß Husens Skulptur „die Ratsherren (...) nicht überzeugt” habe und sie deswegen um einen neuen Künstler in Augsburg nachsuchten. 


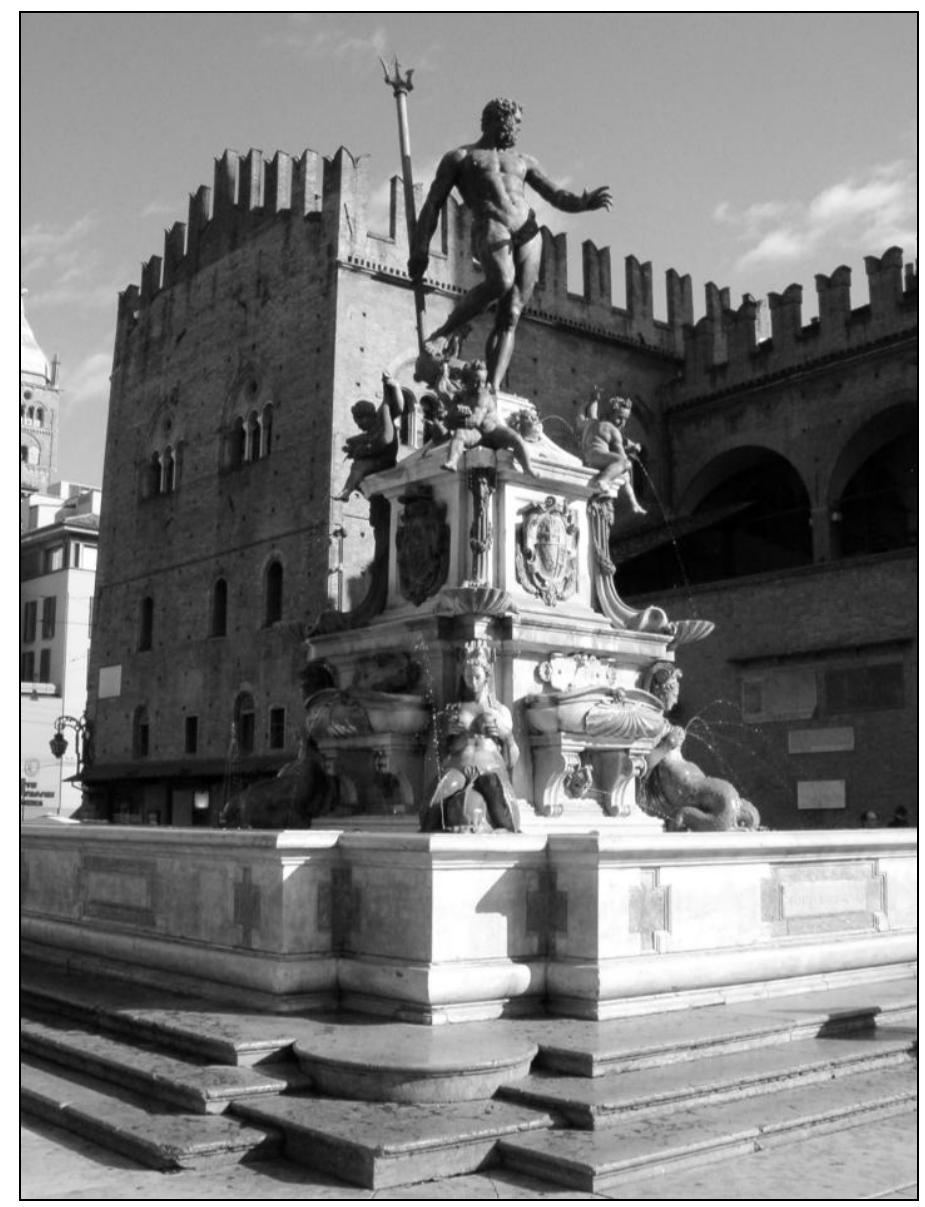

Abb. 2. Giambologna, Neptun, Bologna, Neptunbrunnen

Hier interessiert vor allem die Serie der großen Neptun-Brunnen, die in Florenz und von Florenz ausgehend an anderen Orten Italiens entstanden. Nach dem Tod des zunächst beauftragten Baccio Bandinelli erhielt 1560 Bartolomeo Ammanati im Wettberwerb den Zuschlag für den ersten öffentlichen Monumentalbrunnen der Stadt auf der Piazza della Signoria gleich neben dem Palazzo Vecchio. Anders als die besonders in Gärten und Innenhöfen beliebten Schalenbrunnen, erlaubte das ebenerdige Becken mit zentraler Brunnenstehle eine bis dahin unbekannte Figurengröße. Kurz davor und zugleich entstanden zwei weitere Brunnen dieses neuen Typs: der des Michelangelo-Schülers Giovan Angelo Montorsoli (1557) in Messina (Neptun stehend zwischen Skylla und Charybdis) und Giambolognas Neptunbrunnen in Bologna (vollendet 1565). Sein Bronzeneptun (Abb. 2) machte ihn in ganz Europa berühmt. 
Seine Werkstatt in Florenz zog Mitarbeiter aus vielen Ländern an, die wiederum dazu beitrugen, seine Kunst mittelbar zu verbreiten.

Der Brauch, öffentliche Brunnenanlagen mit antikisierenden Figuren und besonders mit Bronzegüssen zu schmücken, ist schon auf die italienischen Kommunen des Mittelalters zurückzuführen. In Perugia bekrönt den von Nicola und Giovanni Pisani entworfenen, gegen 1278 fertiggestellten Brunnen vor dem Palazzo dei Priori die Gruppe dreier, zu einer Stele verdichteter weiblicher Figuren ${ }^{3}$. Bronzeguß galt den Zeitgenossen als eine eminent antike Technik ${ }^{4}$. Die einheitliche Kleidung der drei weiblichen Figuren, der Peplos, ihre Gestaltung als Karyatiden und das Gefäß, der Kantharos, den sie auf ihren Köpfen tragen, ließen die Gruppe als ein antikes Werk erscheinen. Eine dreigesichtige Statuette, vielleicht eine Darstellung der Hekate, könnte als Vorlage gedient haben. Der mit Reliefbildern und Skulpturen reich verzierte Brunnen memoriert Szenen und Figuren aus der biblischen und der römischen Geschichte, aus dem wirtschaftlichen, politischen und religiösen Leben der Kommune bis hin zu den Porträts der aktuellen Inhaber ihrer beiden höchsten Ämter. Die fingiert antike Bronzegruppe repräsentierte die vorchristliche, etruskische Gründung der Stadt.

Der Bildhauer Lorenzo Ghiberti erzählt in seinen Commentari (um 1450), wie in der Stadt Siena im 14. Jahrhundert bei Fundamentierungsarbeiten eine antike Statue gefunden wurde, auf deren Basis der Name ihres berühmten Meisters, Lysipp, geschrieben stand. Kennern sei die außerordentliche künstlerische Qualität des Werks aufgefallen, und, um es zu würdigen, stellten die Sienesen es auf einen öffentlichen Brunnen - bis sie im Krieg mit den Florentinern große Verluste erlitten. Da sei denselben Sienesen der Gedanke gekommen, ihre gotteslästerliche Idolatrie könne Ursache des Unglücks der Stadt sein, beschlossen, die Statue zu zertrümmern und die Fragmente im Gebiet der Florentiner zu vergraben. Dem Florentiner Ghiberti bietet die Anekdote eine Gelegenheit, Aberglauben und Ignoranz der Sienesen zu verspotten. Seine Beschreibung der Figur - nach einer Zeichnung, die ihm vorliege - ist denkbar knapp, doch handelte es sich wohl um einen Neptun, wie schon Konrad Lange 1879 erkannte: Auf dem Bein, auf das sich die männliche Figur stützte, hielt sie einen Delphin ${ }^{5}$.

${ }^{3}$ Das Original befindet sich heute in der dortigen Regionalgalerie: Perugia, Galleria Nazionale dell'Umbria.

${ }_{4}$ Vgl. N. Gramaccini, Mirabilia. Das Nachleben antiker Statuen vor der Renaissance, Mainz 1996.

${ }^{5}$ L. Ghiberti, I commentari, Hg. O. Morisani, Napoli 1947, S. 56, „ed aveva in sulla gamba in sulla quale ella si posava un alfino". 
Als die Bürger von Augsburg um 1537, das Jahr des großen Bildersturms, die Statue des hl. Ulrich, des Nothelfers gegen Wassernot, von dem öffentlichen Brunnen in der Nähe des Rathauses entfernten und durch einen in Bronze gegossenen Neptun ersetzten, war wiederum der Idolatrie-Vorwurf im Spiel, nun aber in anderer Stoßrichtung. Die Heiligenfigur war zwischen beiden konfessionellen Parteien umstritten. Der mythologische Neptun dagegen stellte einen neutralen Ersatz dar - mit dem Vorzug, zugleich an die römische Gründung der Stadt zu erinnern, deren lateinischer Name Augusta Vindelicorum ihre geographische Lage nach den für sie in vielfacher Hinsicht lebenswichtigen Gewässern vinda und licus (Wertach und Lech) bestimmte ${ }^{6}$. Mit diesen beiden Episoden kommen wir wahrscheinlich den Auffassungen und Absichten nahe, die auch im Fall des Danziger Brunnens zur Wahl des Themas führten: Antikenfiktion und konfessionelle Neutralität.

Handelt es sich nun bei dem Danziger Bronzeneptun lediglich um eine Nachahmung von Artefakten italienischer Herkunft, oder war der Meister enger mit der Weise vertraut, in der diese selbst hervorgebracht wurden? Die Frage nach dem bislang anonymen Urheber der Statue soll im folgenden von der Seite der Bilderfindung her angegangen werden. Dabei wird es nicht genügen, ikonographische Quellen und typengeschichtliche Exempla zu ermitteln. Vielmehr geht es darum, wie der Künstler sich zu den sprachlichen und bildlichen Vorlagen verhielt und eine gegebene Situation, die existierende Platzanlage mit ihren repräsentativen Gebäuden, figürlich ausdeutete. Florentiner Künstler der Renaissance demonstrierten dabei im wesentlichen zwei Haltungen: Gelehrsamkeit im Studium nach der Antike und eigene Beobachtung der phänomenalen Natur. Sie vergewisserten sich der Autorität schriftlicher und visueller Quellen, an welchen Merkmalen ein Thema zu erkennen sei. Sie kompilierten Gestaltelemente aus unterschiedlichen Vorlagen, ergänzten die fragmentarischen oder aus ihrem originären Zusammenhang gelösten Antiken nach ihrer mutmaßlichen Bedeutung. Mehr aber noch kam es den Künstlern der Renaissance darauf an, ein jeweiliges Thema in visueller Evidenz vor Augen zu führen. Nicht sofort mußte sich dem Betrachter die ganze Bedeutung eine Werks in der Anschauung erschließen. Doch verfehlte es sein Ziel, wenn nur ein komplizierter Kommentar es überhaupt zum Sprechen brachte.

${ }^{6}$ Vgl. B. Bushart, Die Augsburger Brunnen und Denkmale um 1600, (in:) Welt im Umbruch. Augsburg zwischen Renaissance und Barock, Ausstellung Katalog, Augsburg, Städtische Kunstsammlungen, Augsburg 1981, S. 82, 85. Zu dem Hans Daucher zugeschriebenen Bronze-Neptun vgl. ibidem, Bd. 2, S. 153-155, Kat. 502. 
Bildende Kunst ist die Darstellung sichtbarer Gegenstände mit sichtbaren Mitteln. Ihr wichtigster Gegenstand aber, wie Leon Battista Alberti (De pictura, 1435) in Anlehnung an antike Autoren betonte, ist der menschliche Leib, sofern dieser immer schon ein darstellender Leib ist. In Haltung und Handlung manifestiert der lebendige Leib Ethos und Pathos. Mimesis, wie sie die Griechen verstanden, hieß zunächst, die sinnlich wahrnehmbare Natur aller belebten und unbelebten Dinge durch Ausdrucksbewegungen des menschlichen Leibes, Stimme und Gebärde, darzustellen. Die Ausdrucksbewegung demonstriere, wie sich ein Ding verhalte. Anders als das Schauspiel, ist die bildende Kunst auf die für sich genommen unbewegte Figur beschränkt. Dabei aber entfaltet sie ihre ganze Illusionskraft, eine prägnante Haltung zu erfinden, die ganz unvermittelt als Äquivalent einer Bewegung und Handlung empfunden wird.

Am Anfang der Florentiner Neptun-Reihe steht Leonardos Beschäftigung mit dem Thema, die wohl in die Zeit seines zweiten Florentiner Aufenthalts nach 1500 fällt. Vasari rühmt in der Vita Leonardos die detailliert ausgearbeitete Zeichnung eines Neptun, welche der Künstler seinem engen Freund Antonio Segni überließ7. Auf dem Blatt sei das aufgewühlte Meer und Neptuns Wagen, gezogen von phantastischen Meerwesen, zu sehen gewesen. Vasari zitiert ein dieser Zeichnung gewidmetes Epigramm: Leonardo habe mit seinem Neptun sogar die Schilderungen Vergils und Homers übertroffen. Der Verbleib des Blattes ist nicht bekannt. Die Nachricht Vasaris genügte, in der Kunst des 16. Jahrhunderts nach sichtbaren Erinnerungen der damals berühmten Neptun-Komposition zu suchen, etwa in der anonymen Zeichnung8 der Accademia Carrara in Bergamo oder in Marcantonio Raimondis Titelkupfer $^{9}$ (Abb. 3) für eine (dann nicht realisierte) Ausgabe von Vergils Aeneis. Solange wir Leonardos Blatt nicht zu sehen bekommen, bleibt das Verhältnis dieser Beispiele zur vermuteten Vorlage unbestimmt ${ }^{10}$.

${ }^{7}$ G. Vasari, Le Vite de' più eccellenti pittori, scultori ed architettori (1568), Hg. G. Milanesi, Bd. 4, Firenze 1906, S. 25; vgl. C. Gould, Leonardo’s „Neptune Drawing”, „Burlington Magazine” 94, 1952, S. 289-294; M. Kemp, Leonardo da Vinci. The marvellous works of nature and man, Oxford 2006, S. 209-210, 266, 319.

${ }^{8}$ Neptun und Seepferde, Rötel auf gelblich grundiertem Papier, 44,5 $\times 51,6 \mathrm{~cm}$; vgl. F. Rossi, I grandi disegni italiani dell'Accademia Carrara in Bergamo, Milano 1985, Abb. 56; C. Gould, op. cit.; L. Nees, Le „Quos Ego” de Marc-Antoine Raimondi, „Nouvelles de l'estampe", 1978, 40-41, S. 18-29.

9 Vgl. K. Oberhuber, The Works of Marcantonio Raimondi and his School, New York 1978 (Illustrated Bartsch, 27), Nr. 352-I.

10 Es ist demnach nicht zulässig, die Zeichnung in Bergamo wie bei Rossi (wie Anm. 7) als „Kopie” nach Leonardo einzuordnen. 


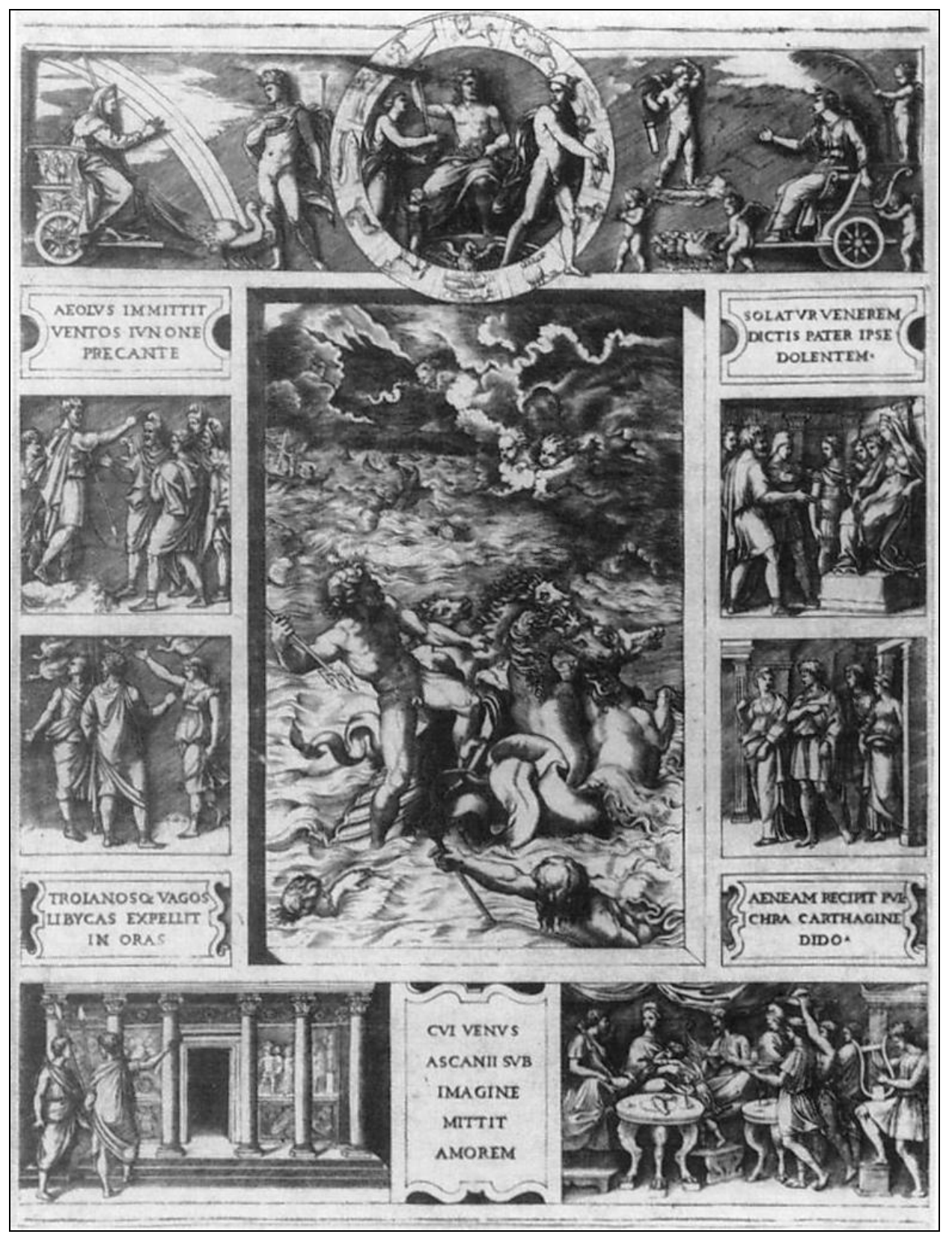

Abb. 3. Marcantonio Raimondi, Titelblatt für Vergils Aeneis, Kupferstich 


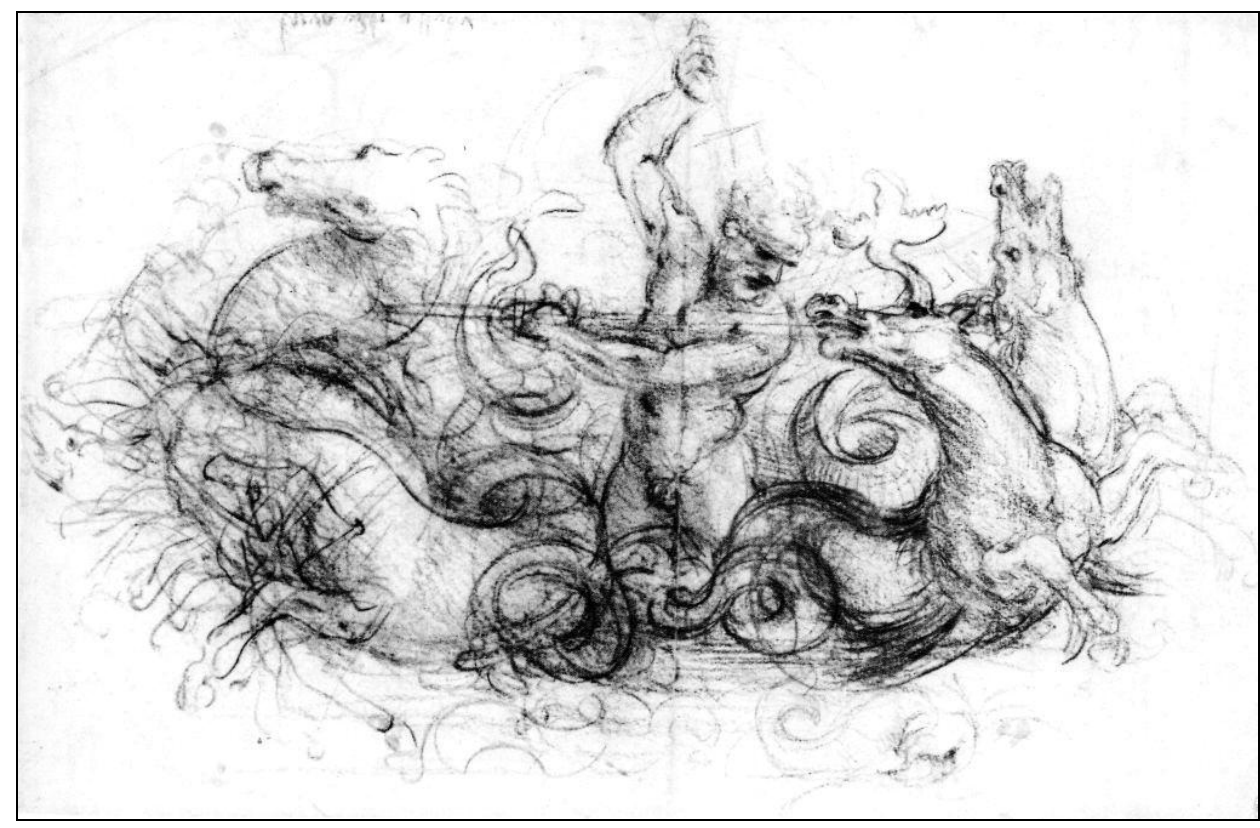

Abb. 4. Leonardo da Vinci, Neptun und Seepferde, Windsor Castle, Royal Library, Inv. 12570

Halten wir uns an eine erhaltene Skizze ${ }^{11}$ (Abb. 4) Leonardos, die stets mit dessen Geschenk an Antonio Segni in Verbindung gebracht wurde. Neptun taucht soeben aus den Fluten auf, den Dreizack schwingend und mit der anderen Hand die sich aufbäumenden Seepferde zügelnd. Wie er fast frontal gesehen ist und die Tiere seiner Quadriga paarweise rechts und links von ihm erscheinen, knüpft der Zeichner vielleicht an den hellenistischen Meerwesensarkophag an, der damals auf den Stufen nach S. Maria in Aracoeli in Rom zu sehen war und von Künstlern studiert wurde ${ }^{12}$. Leonardo löste die Neptun-Gruppe aus der dichten, additiven Bildszene und gewann damit jenen Spielraum eines gleichsam rahmenlosen Bildes, um das Drama von Kraft und Bewegung, von Entfesselung und Zügelung zu entfalten. Betrachten wir die Linea-

11 Windsor Castle, Royal Library, Inv. 12570, Neptun und Seepferde, schwarze Kreide, 25,2 × 38,9 cm; vgl. Leonardo da Vinci Master Draftsman, Hg. C.C. Bambach, New Haven 2003, S. 512-515, Nr. 93; F. Zöllner, Leonardo da Vinci (1452-1519). Dzieła wszystkie, Köln 2006, Abb. 399.

12 Roma, Musei Vaticani. Zum Meerwesensarkophag und seiner Rezeption durch Künstler der Renaissance vgl. P. Pray Bober, R. Rubinstein, Renaissance and Antique Sculpture. A Handbook of Sources, Oxford 1986, S. 131; L. Freedman, Neptune in Classical and Renaissance Visual Art, „International Journal of the Classical Tradition”, 2, 1995, 2, S. 225. 
turen seiner Zeichnung, wie sie aus der Bewegung der zeichnenden Hand heraus, aus Schwüngen und Gegenschwüngen, entwickelt sind, sehen wir die Rosse als Verkörperungen der Wogen und Strudel des bewegten Meeres. Der Kunsthistoriker Cecil Gould bemerkte, daß es Leonardo gefiel, auf wenigstens einem seiner turbulenten Blätter zerstörerischer Orkane und Sintfluten veritable Zephire in den Wolkenmassen figurieren $\mathrm{zu}$ lassen ${ }^{13}$. Leonardo insistiert, nicht nur die Kunst, sondern auch die mythologischen Figuren auf Naturerfahrung zurückzuführen. Die verschlungenen Kurvaturen der Seepferde, die Richtungskontraste der Neptunfigur sind Mimesis der Meeresnatur, wie in den Orkan- und Sintflutbildern die aus den kreisenden Bewegungen der zeichnenden Hand entwickelten Spiralformen die Dynamik des Geschehens und indirekt die darin wirkenden Kräfte und Gegenkräfte illustrieren. Die Kunst will schließlich alles, was auf bloßer Memorierung des schon Gewußten, der Mythologie oder der Dichtung basiert, hinter sich lassen. Dem Betrachter soll nicht nur die Bezeichnung „Neptun” einfallen, er soll vielmehr an den visuellen Qualitäten des Bildes erkennen, wie Neptun sich seiner Natur nach verhalte.

Anders als Leonardos Neptun-Skizze, ist Marcantonio Raimondis Titelkupfer (Abb. 3) zu einer beabsichtigten Ausgabe der Aeneis eindeutig auf Vergils Quos Ego-Episode gemünzt. Juno entfesselte gegen die Flotte der seegeborenen Trojaner einen Sturm. Neptun erscheint machtvoll auf seinem Wagen, entscheidet mit einem knappen Quos ego ${ }^{14}$ („Ich werd' euch ..."), gerichtet gegen die feindlichen Windgötter, die Situation zugunsten der Trojaner. Die nicht zuende gesprochene Proposition ist das sprachliche Äquivalent einer machtvollen Drohgebärde, die Raimondis Kupferstich visualisiert, indem sein Neptun, den Sturmwolken zugewandt, den Dreizack schwingt. In Leonardos Skizze dagegen richtet der Gott den Dreizack nach unten (wie es auch an dem Danziger Neptun auffällt), als wolle er mit einem kräftigen Stoß das Meer aufwühlen, dessen Toben ohnehin die Seepferde in ihrem Ungestüm veranschaulichen ${ }^{15}$.

13 Windsor Castle, Royal Library, Inv. 12376r, Feder über schwarzer Kreide, auf hell grundiertem Papier, $27 \times 40,8$ cm; vgl. F. Zöllner, op. cit., S. 534, Abb. 463. Zu den in der Leonardo-Literatur oft als „Visionen” apostrophierten Texten und Zeichnungen der Orkane und Flutkatastrophen siehe J. Gantner, Leonardos Visionen von der Sintflut und vom Untergang der Welt. Geschichte einer künstlerischen Idee, Bern 1958; A. Marinoni, (in:) Leonardo da Vinci. Scritti letterari, Hg. A. Marinoni, Milano 1987, S. 29-31, 176-183; M. Kemp, op. cit., S. 315-319.

14 Vergil, Aeneis I, $133 \mathrm{ff}$.

${ }_{15}$ M.J. Marek, Ekphrasis und Herrscherallegorie. Antike Bildbeschreibungen im Werk Tizians und Leonardos, Worms 1985, S. 75-104, besonders S. 78, sieht Leonardos verschollene Bilderfindung durch das bei Vasari zitierte Epigramm auf Vergils Quos ego 
Die mythologisch-ikonographischen Handbücher der Renaissance, Lilio Gregorio Giraldis De Deis gentium varia et multiplex historia (erschienen in Basel 1548)16 und nachfolgend Vincenzo Cartaris Le imagini con la spositione de $i$ dei de gli antichi (Venezia 1556 et passim), betonen das wechselnde Erscheinungsbild Neptuns. Er sei von den Alten mal friedlich und ruhig, mal stürmisch bewegt dargestellt worden, wie zuerst bei Homer und dann bei Vergil, dabei unbekleidet, mit dem Dreizack und der Muschel. Cartari fügt hinzu, Neptun zeige sich derart wechselnd, denn gerade so verhalte sich das Meer je nach Wetterlage ${ }^{17}$. Der mimetischen Kunstauffassung korrespondiert eine mimetische Auffassung von den Göttern und ihrer Macht. Das unberechenbar Wechselhafte der Gottheit spiegelt sich in Rosso Fiorentinos Neptun, der uns durch den Stich Caraglios ${ }^{18}$ überliefert ist, und noch in Gianlorenzo Berninis Neptun-Gruppe ${ }^{19}$ (um 1621). Neptun, mit grimmigem Ausdruck, führt den Dreizack, das Ziel seiner Attacke ist nicht zu sehen. Bei Bernini ist dem kämpferischen Habitus ein Triton beigesellt, der in das Muschelhorn bläst. Nicht auf Vergil, sondern Ovid scheint hier angespielt, auf die Episode von Deukalion und Pyrrha ${ }^{20}$. Dort ist es Neptun, der auf Geheiß Jupiters die Sintflut entfesselt, indem er mit seinem Dreizack auf die Erde stößt, so daß ihr die Wasser entströmen, der aber die Sintflut schließlich auch beendet, indem er Triton anweist, in das Muschelhorn zu stoßen und so den Fluten das Signal zum Rückzug zu geben ${ }^{21}$. Neptun ist hier Gebieter des Wassers, nicht nur des Meeres, sondern auch der Quellen. Seine Zuständigkeit für Brunnen und Fontänen ist daraus abzuleiten.

Schon in der hellenistischen Allegorese und ebenso unter dem Vorzeichen des christlichen Weltbildes sind die Götter nur mehr Verkörperungen der Naturkräfte und zugleich der Tugenden, sie zu unterwerfen. Dies macht die Ambivalenz oder Zwiespältigkeit der Götter aus, daß sie Leidenschaft und Ratio zugleich manifestieren. Sie teilen bestimmte Züge mit eben derjenigen Naturgewalt oder dem Element, das zu beherrschen ihnen aufgegeben ist.

bezogen, andererseits findet sie in der erhaltenen Skizze „keine enge Anlehnung an Vergils Text”; M. Kemp, op. cit., S. 319, sowie Leonardo da Vinci..., S. 512, assoziieren bei Leonardos Skizze hingegen Ovid, Metamorphoses I, 283-284, wo Neptun die von Jupiter beschlossene Sintflut entfacht.

${ }^{16}$ L.G. Giraldi, De Deis gentium varia et multiplex historia, Basel 1548, S. 216.

17 V. Cartari, Imagini delli dei de gl'antichi (Venezia 1647), Hg. A. Grossato, Milano 2004, S. 129, „tale si mostra parimente il mare secondo la varietà de’ tempi”.

18 S. Boorsch, J. Spike, Italian Masters of the Sixteenth Century, New York 1978

(Illustrated Bartsch 31), Nr. 28-B; L. Freedman, op. cit., S. 233 ff.

${ }^{19}$ London, Victoria and Albert Museum.

20 C. Avery, Bernini. Genius of the Baroque, London 1997, S. 180-181.

21 Ovid, Metamorphoses I, 283-284, 330-342. 
Vergil nimmt die Quos ego- Episode zum Anlaß, an die Eloquenz eines idealen Herrschers zu erinnern, der die Gemüter der Aufgebrachten alleine schon durch seine Worte befriedet: „ille regit dictis animos et pectora mulcet”22. In humanistischer Deutung verkörperte Neptun Eloquenz und Vernunft ${ }^{23}$. Entsprechend kommentierte Giorgio Vasari in dem ersten seiner Ragionamenti von 1557, einem fiktiven Dialog von Fürst und Maler, daß sein Gemälde des Neptun im Palazzo Vecchio als Allegorie des Fürsten zu verstehen sei24. Es ist Vergils Vision der idealen Herrscherfigur, der sich der Rhetorengestus bei Montorsolis Neptun in Messina und seine fast lässige Variante bei dem Bologneser Giganten verdanken (Abb. 2). Gegenüber der antiken Ikonographie stellt der Rhetorengestus in der Darstellung dieser Gottheit wohl eine Innovation dar, doch kannte bereits die Antike Neptunfiguren als Herrschaftssymbol. Erst die Renaissance scheint sich darauf verlegt zu haben, den Gestus des Dreizackschwingens nicht als beständige, charakteristische Aktionsart der Gottheit, sondern als Illustration einer bestimmten literarischen Episode zu verstehen. Betrachten wir die Situation des Dichters Vergil, drängt sich eine umgekehrte Perspektive auf: Wenn der Gott durch den Gestus charakterisiert ist, konnte der Dichter sich herausgefordert sehen, eine dem durch die Bildwerke verbürgten Charakter gemäße Episode mit Worten auszumalen.

Unter den bildlichen Neptun- oder Poseidon-Exemplaren der Antike ist die eine, alle Aspekte der Danziger Figur vereinende Vorlage nicht nachzuweisen. Wir stoßen vielmehr auf zwei deutlich getrennte Prototypen: Der eine zeigt den Gott in Ruhe, der andere in Bewegung. Dabei ist die Relativität von Ruhe und Bewegung im Medium der bildenden Kunst zu bedenken. Das für sich genommen unbewegte, zeitlich fixierte Bildwerk unterhält eine spezifisch semiotische Beziehung zu zeitlichen Abläufen und narrativen Ordnungen. Wie ist die vorgestellte Dynamik mit der tatsächlichen Statik des Bildwerks vermittelt?

22 Vergil, Aeneis I, 153.

${ }^{23}$ Vgl. R. Preimesberger, Visual Ideas of Papal Authority: The Case of Bologna, (in:) Iconography, propaganda, and legitimation, Hg. A. Ellenius, Oxford 1998, S. 183-186.

${ }^{24}$ G. Vasari, op. cit., Bd. 8, S. 28, „Tutto questo intessuto dell'elemento dell'Acqua, Signor principe mio, è accaduto al duca signore nostro, il quale venuto in aspettamento dal cielo in questo mare del governo delle torbide onde, e fatte tranquille e quiete, per la difficultà di fermare gli animi di questi populi tanto volubili e varj per i venti delle passioni degli animi loro, i quali sono dalli interessi proprj oppressi (...).” Die ragionamenti, für den Druck vorbereitet bereits 1567, erschienen erstmals 1588 bei Filippo Giunti in Florenz. Sie wurden 1619 erneut aufgelegt als Trattato della Pittura. 


\section{DAS RUHEMOTIV: DER HOCHKONTRAPOST - DER FUSS AUF DEM MEERWESEN}

In der antiken Skulptur gibt es drei elementare Darstellungsweisen: erstens die handlungslose, stehende oder thronende Einzelfigur, die, durch Physiognomie, Haltung und Attribute gekennzeichnet, auf die Möglichkeit solcher Handlungen verweist, die eben die dargestellte Gottheit auszeichnen, wie hier im antiken Münzbild einer Sesterze des Agrippa $^{25}$ (Abb. 5) und wieder in der oben genannten Augsburger Brunnenfigur. ${ }^{26}$ Es gibt zweitens vielfigurige, szenische Darstellungen, die die Gottheit in einer bestimmten Episode und Handlung repräsentieren,

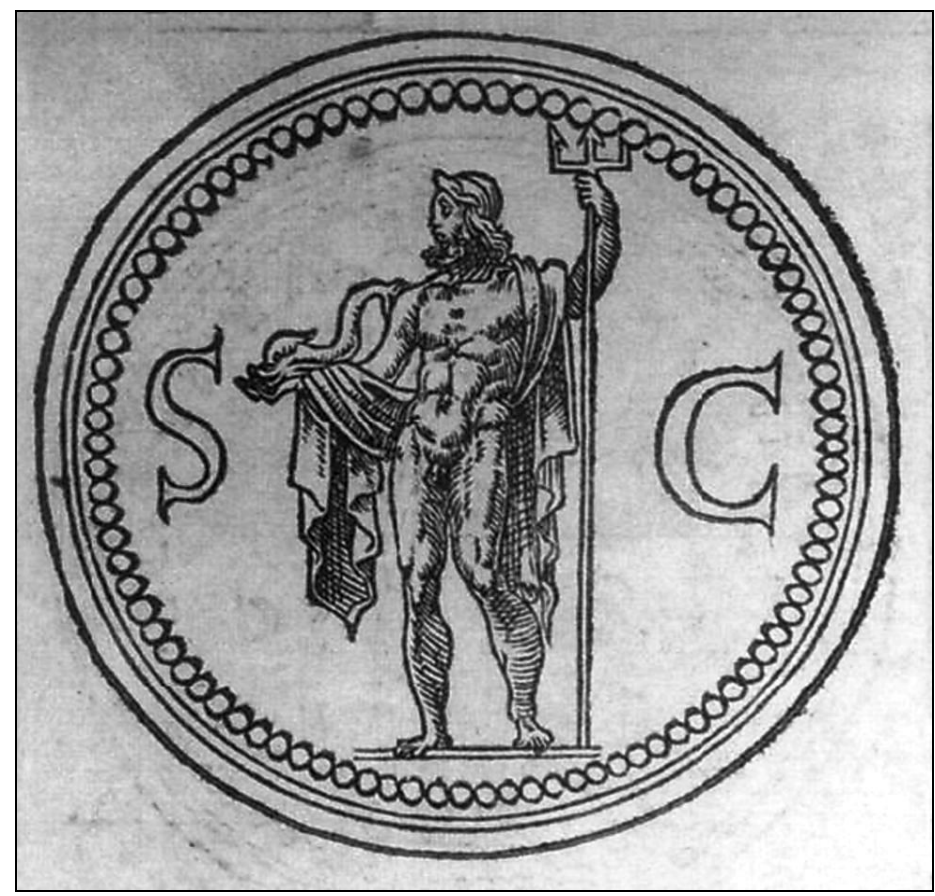

Abb. 5. Neptun, Sesterze des Agrippa, (in:) Guillaume Du Choul, Discorso della Religione antica de Romani (Lyon 1559)

${ }^{25}$ L. Freedman, op. cit., S. 221, Abb. nach Guillaume Du Choul, Discorso della Religione antica de Romani, ins Italienische übertragen von G. Symeoni, Lyon 1559, S. 88.

${ }^{26}$ Dem antiken Münzbild scheint auch der Entwurf Baccio Bandinellis für eine Bronzestatue, die in Genua Andrea Doria als Neptun darstellen sollte, zu folgen. Siehe die Zeichnung in London, British Museum, Andrea Doria als Neptun; abgebildet (in:) J. Poeschke, Die Skulptur der Renaissance in Italien. Michelangelo und seine Zeit, München 1992, S. 167. Der Auftrag wurde dann in den für eine Marmorstatue umgewandelt, die begonnen, aber nicht vollendet wurde. 
und drittens einen Darstellungsmodus zwischen beiden, die isolierte Einzelfigur, die aber in einer bestimmten Aktion begriffen zu sein scheint. Attribute sind dingliche Stellvertreter charakteristischer Handlungen. Dieser dritte Modus „aktiviert” die Attribute ${ }^{27}$. Er zeigt sie in Gebrauch, verkürzt aber die Handlung, die gemeint sein könnte, auf einen bloßen Gestus, was wiederum der stillgestellten Beschaffenheit des Bildmediums entgegenkommt.

Abb. 6. Giambologna, Neptun, Florenz, Museo Nazionale del Bargello

Betrachten wir den Delphin in der Rolle des ,aktivierten” Attributs bei Giambologna. Zunächst dessen Bronze-Neptun in Bologna (Abb. 2). Der Delphin, der das nach hinten ausgestellte Spielbein unterstützt, scheint viel $\mathrm{zu}$ klein, um ernsthaft das Gewicht des Giganten zu tragen, und doch

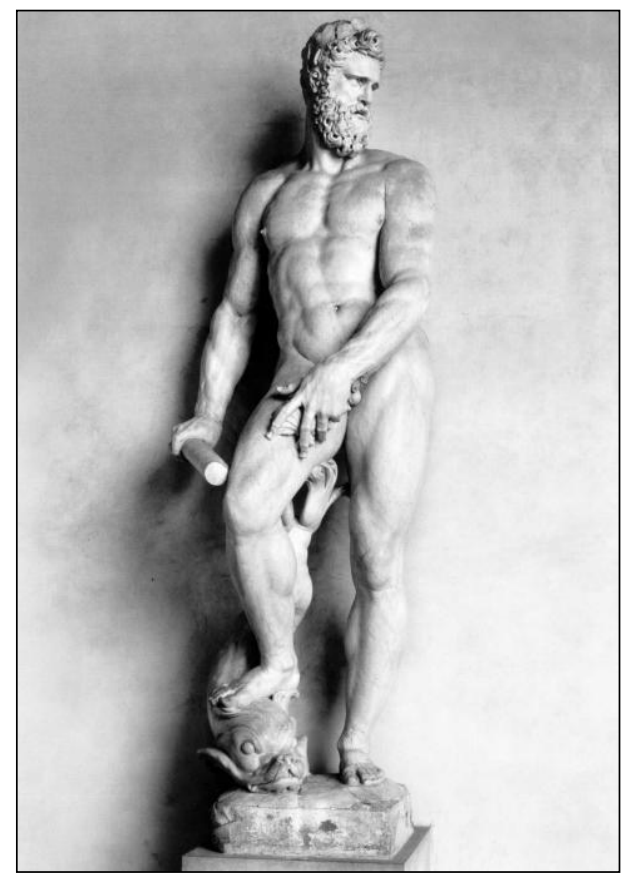
ist es, als steige die soeben eingetroffene Gottheit von ihm herab auf den festen Grund der Plinte ${ }^{28}$. Das andere Beispiel ist Giambolognas marmorner Oceanus-Neptun (Abb. 6). Er bekrönte seit 1574 in der vom Palazzo Pitti, der herzoglichen Residenz, ausgehenden Sichtachse der Boboli-Gärten den seinerzeit wohl

27 Zum Begriff des „aktivierten” Attributs vgl. M. Campbell, Giambologna’s Oceanus Fountain. Identifications and Interpretations, (in:) Boboli '90, Atti del Convegno internazionale di studi per la salvaguardia e la valorizzazione del giardino (Firenze, 9-11 marzo 1989), Hg. C. Acidini Luchinat, E. Garbero Zorzi, Bd. 1, Firenze 1991, S. 98.

${ }_{28}$ B. Laschke, Un ritratto di Giovanni Bologna e la Fontana di Oceano nel Giardino di Boboli, (in:) Giambologna tra Firenze e l'Europa, Firenze 2000, S. 69, bezieht die Stellung der Beine der Neptun-Figur auf die Quos-Ego-Episode: Der Gott steige von dem Delphin, seinem mythologischen Reittier, und mache Anstalten, die aufgeregten Wogen des Meeres zu beruhigen. 
größten Schalenbrunnen Europas ${ }^{29}$. Dieser wurde 1618 in die Anlage des sogenannten Isolotto im neu gestalteten westlichen Teil des Gartens transferiert. Der rechte Fuß der Gottheit ruht auf dem Delphin.

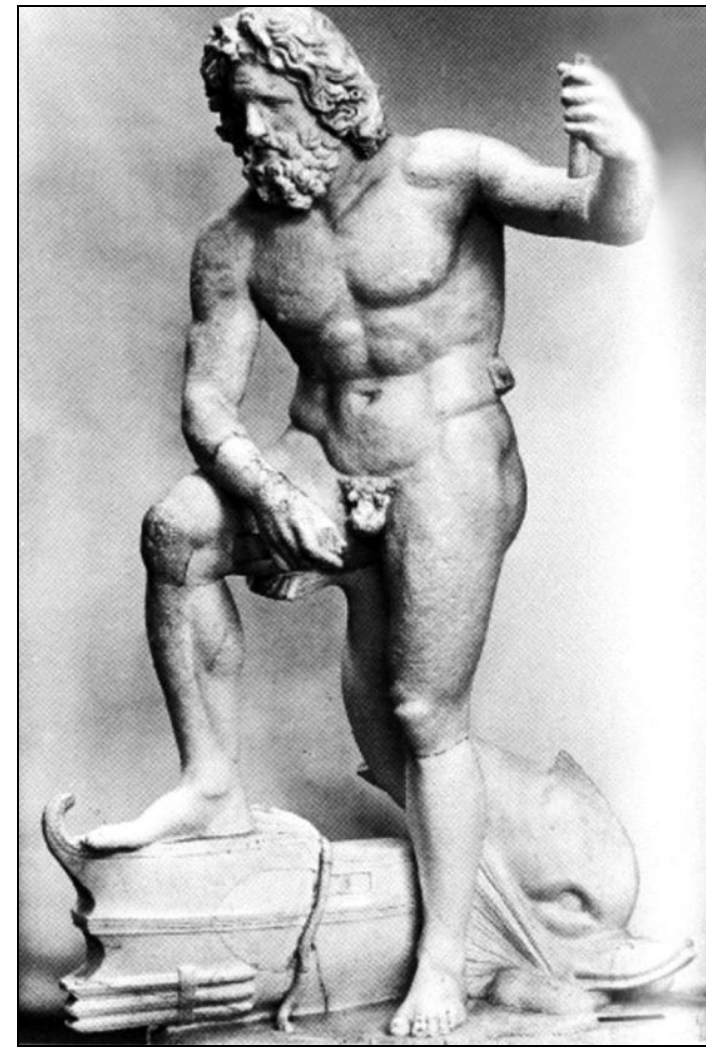

Abb. 7. Neptun, Rom, Vatikan, Museo Gregoriano Profano

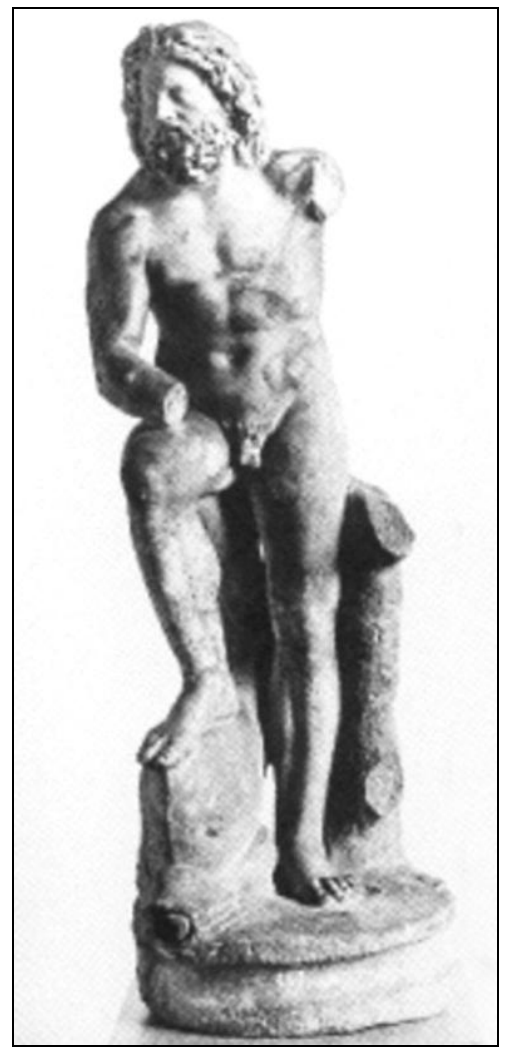

Abb. 8. Neptun, Dresden, Staatliche Kunstsammlungen, Skulpturensammlung

Der Hochkontrapost, das angezogene, erhöht aufgestellte Bein, gilt als Erkennungszeichen besonders der reifen und späten Werke Giambolognas ${ }^{30}$. Leicht wird dabei übersehen, das beide Neptun-Figuren Giambolognas, soweit der Delphin in der Rolle der Fußstütze auftritt, auf antike, ikonographisch relevante Vorbilder rekurieren. Der aufgestützte $\mathrm{Fuß}$ ist zunächst ein Ruhemotiv. Und in dieser Akzentuierung begegnet es auch in der antiken Poseidon-Neptun-Ikonographie als Lateran-Ty-

${ }^{29}$ Vgl. M. Campbell, op. cit., S. 92 ff. Durchmesser der monolithischen Granitschale: $7,58 \mathrm{~m}$. Neptun/Oceanus ist umgeben von den drei Personifikationen der Flüsse Nil, Ganges und Euphrat.

30 So bei F. Kriegbaum, op. cit., S. 242. 
pus, benannt nach einer in der frühen Neuzeit beim Lateranspalast in Rom aufgestellten Kopie der antoninischen Zeit (Abb. 7), heute in den vatikanischen Museen, nach einem hellenistischen Original aus dem Umkreis des Lysipp ${ }^{31}$. Der stehende Poseidon setzt den (in der Regel rechten) Fuß erhöht auf, lehnt den Arm derselben Seite auf den Oberschenkel und stützt die andere (in der Regel linke) Hand auf den Dreizack. In Varianten des Typus begegnet an Stelle des Schiffsmodells ein mehr oder weniger verkleinerter Delphin - so bei einer Bronzestatuette $^{32}$ in Venedig oder einer hellenistischen Marmorstatue ${ }^{33}$ in Dresden (Abb. 8).

Der Archäologe Konrad Lange - in einer Publikation von 1879 rekonstruierte aus den Varianten dieses Typus das originäre Aussehen einer verlorenen Bronzestatue des Lysipp, die an prominentem Ort, im Poseidon-Tempel von Isthmia bei Korinth, als Kultbild gedient habe ${ }^{34}$. Anders als bei den hier gezeigten Beispielen habe dieser Poseidon seinen Fuß auf einen Felsen gesetzt und in der rechten, auf das Knie gelehnten Hand den Delphin gehalten. Der Archäologe dachte sich die Darstellung durchaus unter einem narrativen Aspekt, nämlich als Ruhe „nach vollendeter Arbeit“35, analog zum Herkules desselben Lysipp, dessen Typus vor allem durch den Herkules Farnese berühmt wurde. Ein „schlüpfriger Delphin als Unterlage des aufgestützten Fußes" schien dagegen zu der entschiedenen Ruhepose der Poseidon-Figur nicht zu passen, zu der auch gehörte, den Dreizack in die Linke zu geben (den aktivierten Dreizack bewegt Poseidon stets mit der rechten Hand). Eine „so unverständige Verwendung des Delphins“, den Fuß der Gottheit zu stützen, wollte Konrad Lange einem griechischen Künstler der Klassik nicht zutrauen ${ }^{36}$. Immerhin aber scheint es eben dieses Motiv dann bereits in hellenistischen Skulpturen zu geben.

Was Konrad Lange paradox erschien, verrät seinen Sinn, wenn wir uns die notorische Instabilität des Elements vergegenwärtigen, welches Poseidon repräsentiert und beherrscht. Der Fuß auf dem Delphin deutet die Epiphanie der Gottheit an, als sei sie soeben in Begleitung und mit Hilfe des Delphins erschienen und regelrecht aus den Fluten aufgetaucht. Allgemein gehören Fußtrittmotive in zwei verwandte Kontexte.

${ }^{31}$ Roma, Museo Gregoriano Profano, Inv. 10315; vgl. E. Simon, Poseidon, (in:) Lexicon iconographicum mythologiae classicae, Bd. 7, 1, Zürich 1994, S. 452 ff., 486.

32 Venezia, Museo Archeologico; vgl. E. Simon, Poseidon..., S. 486.

${ }^{33}$ Dresden, Staatliche Kunstsammlungen, H. 157 cm; E. Simon, Poseidon ..., S. 452 ff.

${ }^{34} \mathrm{~K}$. Lange, Das Motiv des aufgestützten Fußes in der antiken Kunst und dessen statuarische Verwendung durch Lysippos, Leipzig 1879, S. 31-52.

35 Ibidem, S. 51.

${ }^{36}$ Ibidem, S. 35. 
In Zweiergruppen zeigen sie den Triumph über einen unterlegenen Gegner an. Sie können aber auch das Verhältnis von Herrschen und Dienen veranschaulichen. Der Delphin ist hier nicht Gegner, sondern Diener der Gottheit.

Pausanias in seiner Beschreibung Griechenlands erwähnt auf der Agorà in Korinth einen Brunnen, bekrönt von der Bronzestatue des Poseidon mit einem wasserspeienden Delphin unter den Füßen ${ }^{37}$. Bei einer hellenistischen, aus Rom importierten Marmorstatue in Dresden, wiederum des Lateran-Typus, ist der Delphin mit einer Röhre ausgestattet, so daß Wasser aus seiner Schnauze entspringen konnte. Ganz in diesem Sinn wurde noch Baccio Bandinellis Ehrenstatue für Andrea Doria als Neptun, bestellt von der Republik Genua, entstanden zwischen 1534 und 1538 und wegen Streitigkeiten mit den Genuesen unvollendet geblieben, schließlich als Brunnenfigur auf der Piazza del Duomo in Carrara installiert ${ }^{38}$.

Wenn es stimmen sollte, daß der Hochkontrapost des PoseidonNeptun in der Antike originär zu einem Ruhemotiv gehört, dann knüpfte Giambologna doch an solche hellenistische Varianten an, die ihn bereits mit einem „aktivierten“ Attribut verbinden. Der Dreizack seines Bologneser Kolosses, wenn auch mit der Rechten, d.h. der üblichen Aktionshand, gehalten, bleibt dennoch wie bei dem Lateran-Typus, in Ruhe. Der lässige, mit dem linken Arm ausgeführte Rhetorengestus unterstreicht die durch nichts zu beunruhigende Souveränität der Gottheit.

\section{DAS BEWEGUNGSMOTIV: DIE ATTACKE MIT DEM DREIZACK}

Wiederum gilt es, antike Prototypen und ihre Anverwandlung durch die Künstler der Renaissance in Betracht zu ziehen. Eine hellenistische Bronzestatuette ${ }^{39}$ (Abb. 9) zeigt offenbar Poseidon, wie er den (verlorenen) Dreizack mit der Rechten einsetzt, während in seiner Linken wahrscheinlich ein Delphin lag. Entweder gehörte die Statuette zu einer Gruppe, die neben der Gottheit einen Giganten umfaßte, dem der Angriff galt, oder die Gottheit stieß mit dem Dreizack gegen einen Felsen, um eine Quelle entspringen zu lassen ${ }^{40}$. Poseidon konnte aber auch losgelöst

37 Pausanias, Graeciae descriptio 2, 2, 8.

${ }^{38} \mathrm{Zu}$ Bandinellis unvollendeter Statue als Brunnenfigur vgl. B. Laschke, op. cit., S. 79, Abb. 14.

39 Paris, Louvre; vgl. E. Simon, Poseidon..., S. 452, Nr. 29.

40 W. Fuchs, Die Skulptur der Griechen, München 1993, S. 135, versteht den Gestus so, daß Poseidon den Dreizack in einen Felsen stoße „um eine Quelle zu schaffen. In seiner 
Abb. 9. Neptun / Poseidon, Paris, Musée du Louvre

von einem bestimmten narrativen Kontext in dieser Aktion fixiert werden, die für ihn so charakteristisch war wie für Zeus das Schleudern der Blitze, Athena Promachos das Schwingen der Lanze oder Apollo das Verschießen der Pfeile ${ }^{41}$. Eben so reduziert ist der Meeresgott und Erderschütterer seit archaischer Zeit auf den Münzen (Abb. 10) seiner Stadt Poseido-

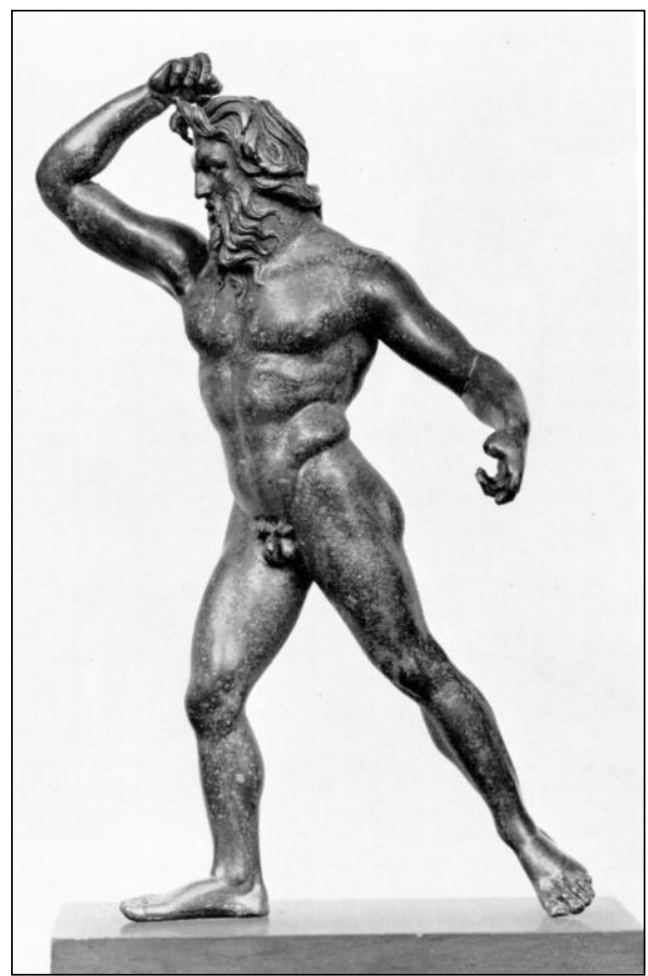
nia (Paestum) dargestellt ${ }^{42}$. Die Handlung konnte in vielteilige szenische Bilder übernommen werden. Poseidon-Neptun erscheint stehend und den Dreizack schwingend inmitten seines Seepferdegespanns auf dem genannten Meerwesensarkophag. In Leonardos da Vinci Skizze (Abb. 4), die sich vielleicht an diesem oder einem unbekannten gleichartigen Vorbild orientierte, ist ebenfalls kein bestimmtes Ziel der Attacke des Gottes zu erkennen.

Die Vita Giambolognas weiß zu berichten, daß auch er sich an dem Florentiner Wettbewerb von 1560 für den Neptun-Brunnen auf der Piazza della Signoria beteiligte. Sofern sein Bologneser Bronzeneptun rasch darauf folgte, hält sich in der aktuellen Diskussion die Vermutung, daß er sein zurückgewiesenes Projekt wenigstens teilweise für den Brunnen in Bologna verwendete ${ }^{43}$. Alleine schon durch die Chronologie wurde er

Linken lag wohl ein Delphin“. Dagegen E. Simon, Poseidon ..., S. 452, Nr. 29, die Gottheit steche mit dem Dreizack vielleicht auf einen Giganten (bei Poseidon meist Polybotes).

${ }^{41}$ E. Simon, Die Götter der Griechen, München 1998, S. 58.

42 Ibidem, S.79; E. Simon, Poseidon..., Bd. 7, 2, S. 454, Taf. 357, Nr. 61-63.

${ }^{43}$ Giambologna. Sculptor to the Medici (1529-1608), Ausstellung Katalog, Royal Scottish Museum Edinburgh, Victoria and Albert Museum London, Kunsthistorisches Museum Wien, Hg. C. Avery, A. Radcliffe, Edinburgh 1978, S. 291; R. Preimesberger, op. cit., S. 183. 


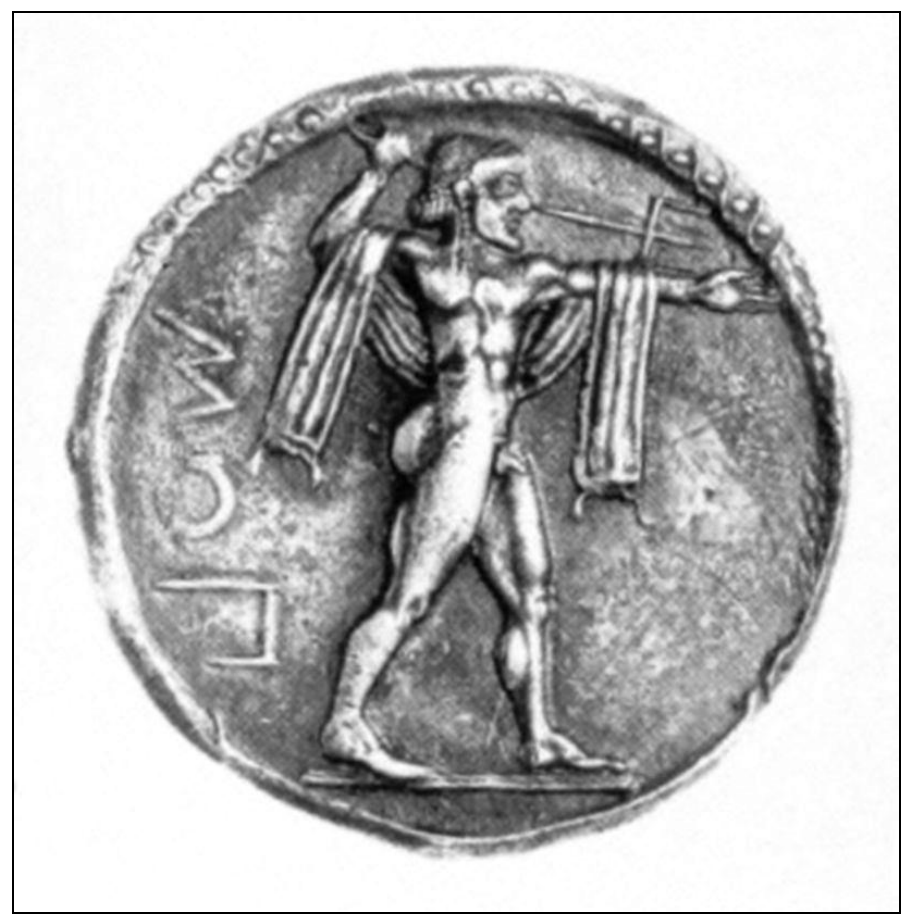

Abb. 10. Poseidon, Münze der Stadt Poseidonia (Paestum)

zu einer Konkurrenz für die größere und langsamer entstehende Florentiner Anlage. Eine Skizze44 (Abb. 11) Bartolomeo Ammanatis für das Florentiner Vorhaben kommt dem näher, was auch Giambologna bei seinem raumgreifenden Bronzeneptun beschäftigte. Wie bei Giambologna steht Neptun auf einem hohen, architektonisch gestalteten Sockel. Wenn sich Ammanatis Figur mit dem linken Knie auf zwei verschlungene Delphine stützt, erinnert dies an Michelangelos unvollendete Allegorie des Sieges, die erst 1565 im Salone dei Cinquecento im Palazzo Vecchio aufgestellt wurde ${ }^{45}$. An der Stelle des unterlegenen Gegners in Michelangelos allegorischer Gruppe erscheinen in der Skizze die Meerestiere, die die Gottheit herbeitrugen. Sie sind - wie bei Giambologna - aktiviert. Mehr noch aktiviert der Zeichner den Dreizack. Sofern dieser Neptun den Kopf seitwärts wendet, als blicke er auf ein entferntes Ziel, könnten wir die Szene als Drohgebärde in Analogie zur Sprachgebärde des Quos ego verstehen. Ammanati realisierte auf der Piazza della Signoria schließlich eine niedriger aufgestellte und beruhigte Standfigur mit blockhaft ge-

${ }^{44}$ London, Privatsammlung; vgl. J. Poeschke, op. cit., S. 204.

45 Ibidem, S. 102-104. 
schlossener Silhouette, wodurch er offenbar Risiken der Marmorbearbeitung umging und den schon durch Baccio Bandinelli vorbereiteten Block respektierte ${ }^{46}$.

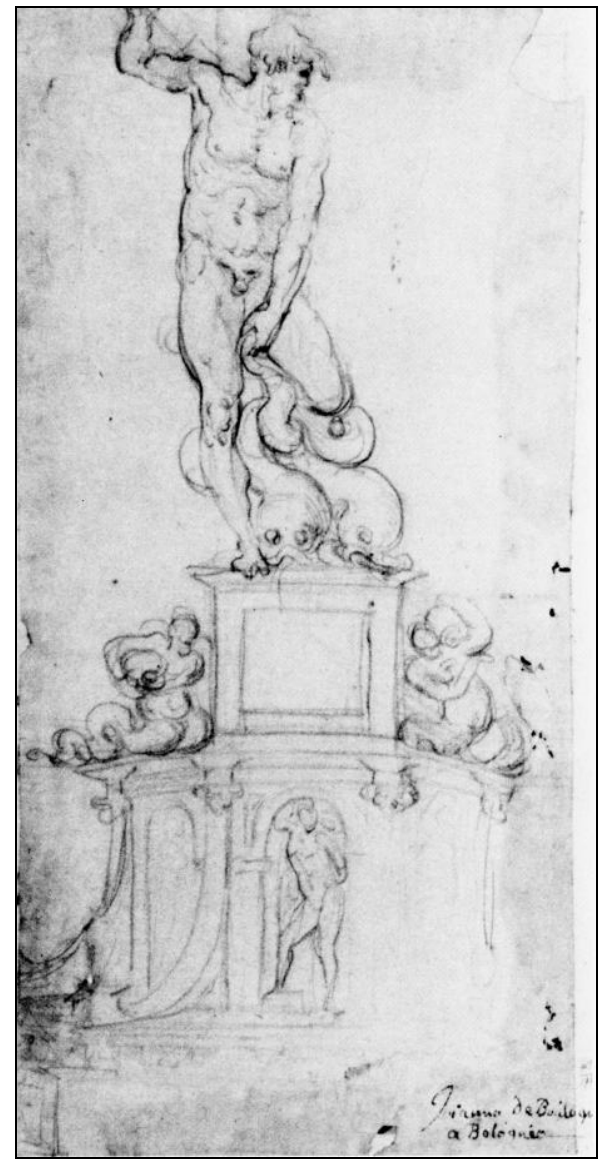

Abb. 11. Bartolomeo Ammanati, Neptunbrunnen, London, Privatsammlung

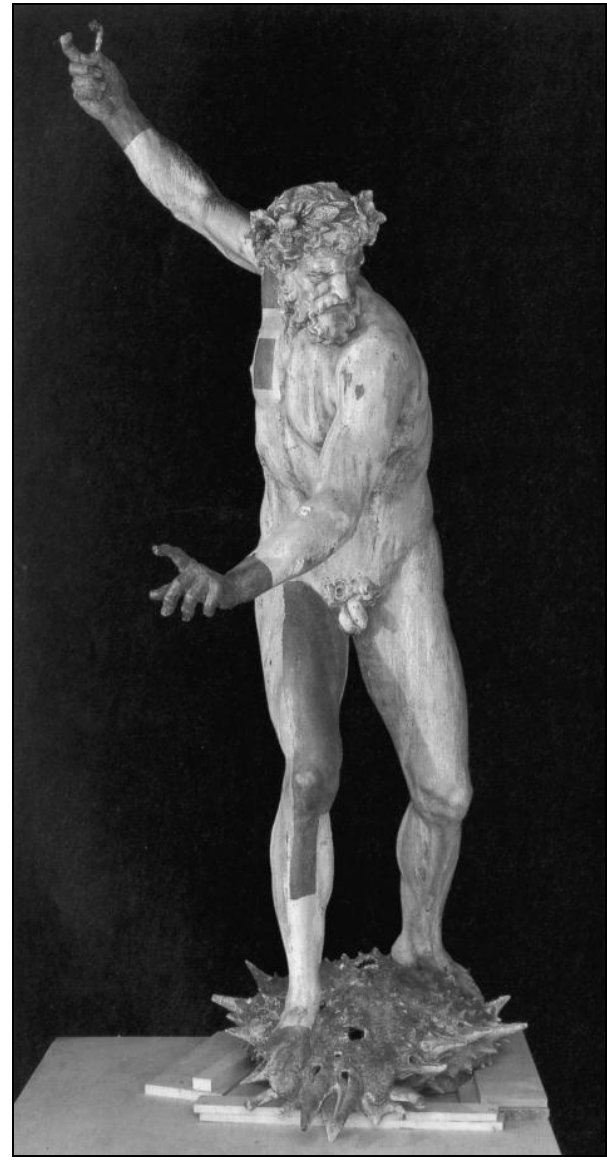

Abb. 12. Stoldo Lorenzi, Neptun, Florenz, Boboli-Gärten

Nicht die bloße Drohgebärde, sondern die - ganz im Sinn antiker Vorbilder - objektlose Handlung stellte wenige Jahre später ein anderer

46 Ammanati realisierte für den Brunnen der Piazza della Signoria schließlich eine niedriger aufgestellte und größer bemessene beruhigte Standfigur in geschlossenerer Silhouette, die eher einem anderen Vorbild Michelangelos, dessen Marmordavid, folgt. Raffaele Borghini (Il riposo, Firenze 1584) verteidigte den Künstler, daß der schon in seinen Maßen fertigte Block, den Baccio Bandinelli hinterließ, für Ammanatis ursprünglichen Plan, eine Figur mit angehobenen, vom Körper gelösten Armen zu schaffen, keine Substanz geboten habe, vgl. J. Poeschke, op. cit., S. 206. 
Florentiner Neptunbrunnen dar. Im Jahr 1566, aus Anlaß der Vermählung von Prinz Francesco de' Medici und Johanna von Österreich, wurde in Florenz ein Maskenumzug veranstaltet, der die Genealogie der antiken Götter zum Thema hatte. Einer der Festwagen zeigte den Triumph Neptuns, der Herzog Cosimo I. so sehr gefiel, daß er, wie Vasari berichtet $^{47}$, den Bildhauer Stoldo Lorenzi da Settignano (1534-1583) mit den Figuren beauftragte, die seit 1574 einen weiteren großen Schalenbrunnen in der Sichtachse der Boboli-Gärten schmückten ${ }^{48}$. Dieser Brunnen stand bei dem großen Fischteich, „vivaio grande”. Um 15951597 wurde er an die Nordseite des Palastes transloziert. Zwischen 1633 und 1636 kehrten die Figuren an ihren ersten Ort zurück, wobei die Brunnenschale durch einen künstlichen Felsen inmitten eines runden Bassins abgelöst wurde. Daß dieser Neptun von Anfang an mit der linken Hand zusätzlich den Schaft des Dreizacks führte (Abb. 12), wie er wenigstens seit der Neuaufstellung im 17. Jahrhundert montiert war, scheint mir nicht plausibel. Der nach links quer vor der Brust gestreckte Arm veranschaulichte vielmehr, daß er wie ein Diskobolos Schwung zu nehmen hat für die folgende Bewegung, bei der sich der Leib auch um die eigene Achse drehen würde. Wenn er nach unten zielt, wo wir uns das Fischbecken denken müssen, erinnert er an die originäre Funktion des Dreizacks als eine Harpune mit Widerhaken, wie sie beim Fischfang verwendet wurde 49 .

In dem Danziger Neptun finden wir eine ähnliche Ausgangslage und einen ebenso vorgestellten Bewegungsablauf. Wiederum kommt es auf den Blick über die vorgezogene Schulter an, der die Richtung des nachfolgenden Dreizackstoßes anzeigt. Wiederum ist das rechte Bein das Standbein. Anders jedoch als bei Lorenzis Figur zielt Neptun (für den Betrachter) nach links. Die bevorstehende Drehung des Rumpfes erfordert, das linke Bein anders zu plazieren, es nämlich nach vorne zu stellen und seitlich aufzustützen, so daß es den Belastungswechsel auffangen könnte. Dem genügt das vorliegende Fußtrittmotiv, das zugleich an den antiken Typus des hochgestellten Fußes anknüpft. Die Figur wirkt keineswegs so „verkrampft”, wie Helmut Carl meinte ${ }^{50}$. Sie ist vielmehr in der Anspannung, die sie veranschaulicht, und in der Aktion, die aus ihr zu folgen scheint, plausibel. Die von dem Hochkontrapost

47 G. Vasari, op. cit., Bd. 7, S. 637.

48 Zur Gestalt und Geschichte dieses Brunnens vgl. C. Acidini Luchinat, La fontana del Nettuno, viaggi e metamorfosi, (in:) Boboli '90..., S. 31-45; M. Chiavacci, (in:) L.M. Medri, Il giardino di Boboli, Milano 2003, S. 190.

49 E. Simon, Die Götter..., S. 69.

50 H. Carl, op. cit., S. 163-165. 
ausgehende Drehung um die Körperachse und die Gegendrehung des Oberkörpers entsprechen dem Konzept, das die moderne Stilkritik in Anlehnung an das Vokabular des Cinquecento als figura serpentinata ${ }^{51}$ bezeichnet. Sie scheint den Betrachter selbst in Bewegung versetzen zu wollen, so daß er, den Richtungsvektoren der Figur folgend, sie umschreite. Zugleich erlaubt sie, die vertikale Dimension als Bewegungsraum mitzuerleben.

Was Giambologna betrifft, ist seine Verwendung des Hochkontraposts bereits an der Personifikation der Florentia zu erkennen, deren $\mathrm{Gu} ß$ der junge Giambologna laut Vasari nach einem Terracotta-Modell anfertigte, das der Bildhauer Niccolò Tribolo 1550 bei seinem Tod hinterlassen hatte ${ }^{52}$. Die Figur bekrönte einen zunächst für die Villa Medici in Castello geschaffenen, dann nach der Villa Petraia transferierten Kandelaber-Brunnen. Ihr nächstes Modell hat diese Pose in Skulpturen des Hochhellenismus. Am Torso der Terrakotta-Statuette ${ }^{53}$ einer Aphrodite in Berlin ist abzulesen, daß das linke Bein hoch aufgestützt war. Auf die so eingeleitete Drehung der Hüfte antwortet die Gegendrehung des Oberkörpers ${ }^{54}$.

Das Original des Oceanus-Neptun Giambolognas ist heute in Florenz im Museo del Bargello zu sehen. Im selben Museum befindet sich Michelangelos unvollendeter Marmor-Apollo (1525-1530), der wiederum eine Figurenerfindung 55 Michelangelos aus dem frühen, nicht realisierten

51 Zur Herkunft des Terminus bei Lomazzo und seiner Verwendung in der Kunstkritik vgl. David Summers, Maniera and Movement: the Figura Serpentinata, „Art Quarterly“ 35, 1972, S. 269-301; ferner Michael Cole, The Figura Sforzata: modelling, power and the Mannerist body, „Art History”, 24, 2001, 4, S. 520-551.

52 Vgl. C. Avery, Florentine Renaissance Sculpture, London 1970, S. 213.

${ }^{53}$ Berlin, Staatliche Museen, Antikensammlung; vgl. W. Fuchs, op. cit., S. 233, Abb. 252.

54 Die von Niccolò Tribolo entworfene Florentia ist, nach ihrem antiken Typus, eine Venus-Aphrodite Anadyomene. Bemerkenswert ist, daß diese bewegte Pose in der Antike zuerst bei weiblichen Figuren auftaucht, die ihre leibliche Schönheit zur Schau stellen. Aphrodite, die den Schild des Ares hält, um sich darin zu spiegeln, ist vor allem bekannt durch die Marmorkopie aus Capua nach einem spätklassischen Bronzeoriginal, das vielleicht als Kultbild des Aphrodite-Tempels von Korinth diente. Der linke Fuß ruht erhöht auf dem Helm des Ares, daran erinnernd, daß Aphrodite mit den Waffen ihrer Schönheit den Kriegsgott entwaffnete. Der Hochkontrapost der Aphrodite aus Capua und die Richtungskontraste der Berliner Aphrodite liegen offenbar weiteren Figurenerfindungen des italienischen 16. Jahrhunderts zugrunde - von der berühmten Galatea Raffaels in der Villa Chigi bis hin zu der Ceres Hubert Gerhards 1584-1587 am Wittelsbacherbrunnen in München.

55 Vgl. die Skizze für die Figur eines David auf dem Blatt in Paris, Louvre, Inv. 714r., Feder in Braun, 26,2 × 18,5 cm; C. De Tolnay, Corpus dei disegni di Michelangelo, Bd. 1, Novara 1975, Kat. 19. 
Auftrag um 1501 eines Bronze-David aufgreift ${ }^{56}$. Apollo setzt den rechten Fuß auf den Sonnenball (Abb. 13), und wir erkennen daran die Sphäre, deren Herrscher er ist. Die Gottheit ist im Begriff, mit der Linken einen Pfeil aus dem Köcher zu ziehen. Über die vorgezogene Schulter blickt sie herab, das Ziel zu erspähen. Das hochgestellte Bein bereitet zwanglos die Drehung des Rumpfes vor, auf die eine Wendung des Kopfes in entgegengesetzte Richtung antwortet. Ganz unmittelbar stellt sich der Betrachter diesen Apollo an einem sehr erhöhten Ort und das imaginäre,

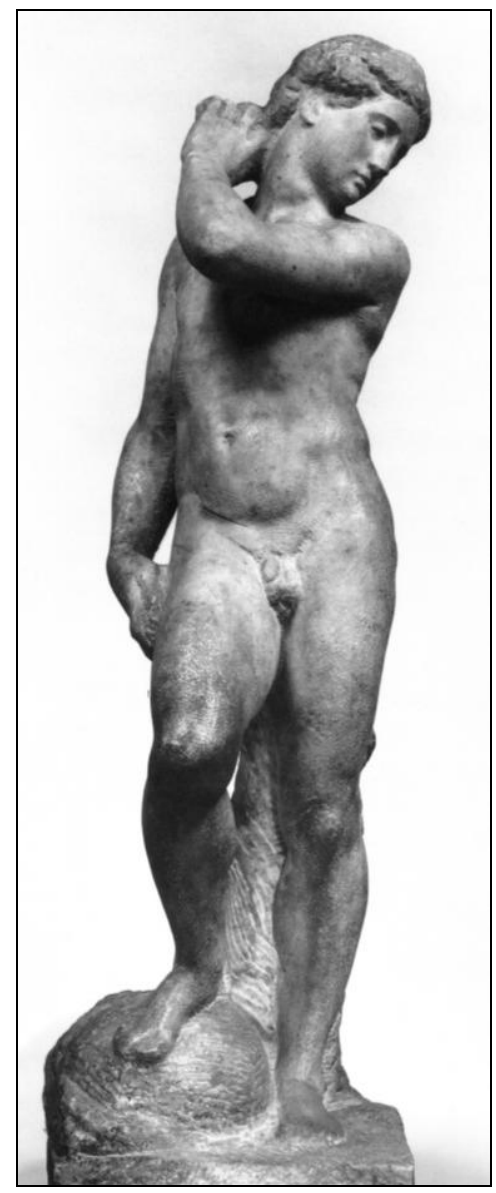

Abb. 13. Michelangelo Buonarroti, Apollo, Florenz, Museo Nazionale del Bargello

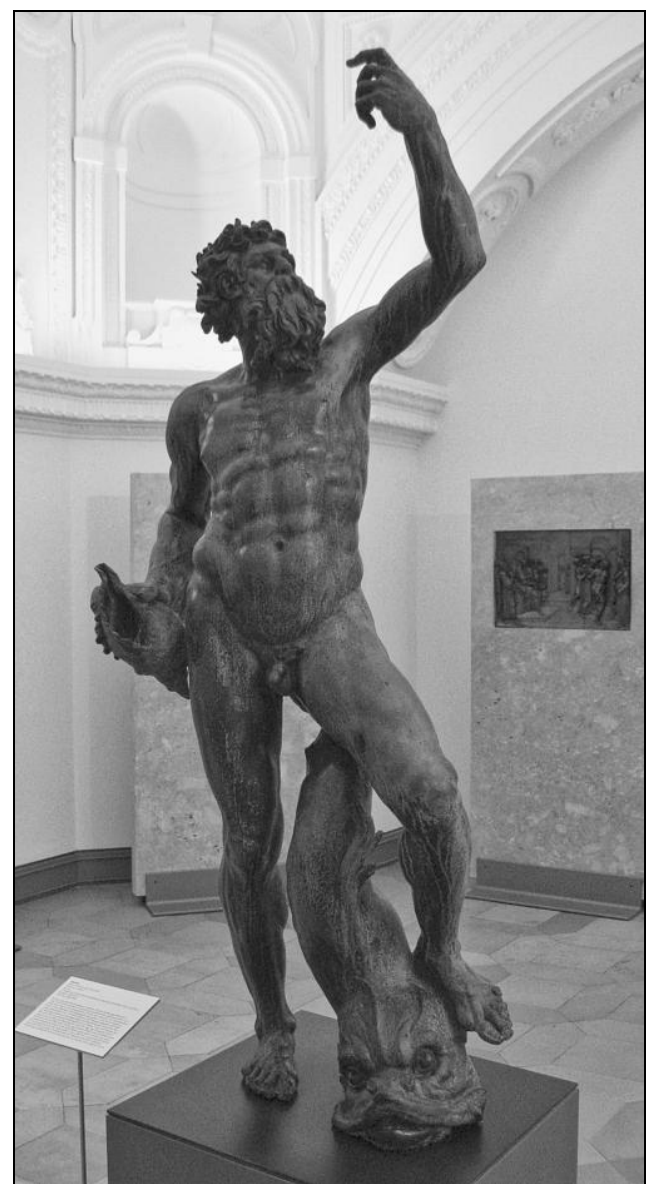

Abb. 14. Hubert Gerhard, Neptun, München, Bayerisches Nationalmuseum

${ }^{56}$ Dort war der Hochkontrapost mit dem Fußtrittmotiv verbunden, das den Sieg über Goliath veranschaulicht: David setzt den Fuß auf das am Boden liegende, abgetrennte Haupt des Philisters. 
unsichtbare Opfer des sicher tödlichen Schusses tief unten vor ${ }^{57}$. Ähnlich blickt der Danziger Neptun über die vorgezogene rechte Schulter auf ein unsichtbares Ziel, das sich in erreichbarer Nähe unten zu seinen Füßen befinden müßte. Dargestellt ist, wie in der antiken Plastik, die Epiphanie der Gottheit. Sie ist soeben aus den Fluten aufgestiegen, erkennbar auch an dem naturalistischen Detail der gekräuselten bronzenen Oberfläche der Seepferde, als sähen wir hier das Wasser gerade von ihren Leibern rinnen.

Der Danziger Neptun manifestiert eine so genaue Kenntnis des Florentiner Entwurfsverfahrens und ihres Antikenbezugs, daß wir den Urheber der Gußvorlage in der Reihe der dort ausgebildeten Künstler suchen müssen ${ }^{58}$. Isaak van den Blockes zentrales Ovalgemälde von 1608 der Decke des Roten Saales ${ }^{59}$ im Rathaus der Danziger Rechtsstadt zeigt auf dem Platz vor dem Artushof einen Brunnen, dessen Gestalt der später realisierten weitgehend entspricht ${ }^{60}$. Nur die bekrönende Figur des Neptun ist deutlich verschieden - und doch so charakterisiert, daß wir das oben beschriebene Feld antiker und nachantiker Hochkontrapostfiguren nicht verlassen. Im Gemälde stützt sich Neptun mit der seitwärts in die Höhe gestreckten Linken auf den aufgestellten Schaft des Dreizacks. Neben dem erhöht aufgesetzten rechten Fuß ist der Kopf eines Fischs (Delphins?) zu erkennen, der als Wasserspeier fungiert. Unklar scheint lediglich, welcher Gegenstand auf dem rechten Unterarm liegen soll (ein kleiner Delphin, ein Muschelhorn?).

57 Der Florentiner Francesco Salviati (1510-1563) zeichnete nach Michelangelos unvollendeter Figur einen Apollo, der nun allerdings auf dem von Pfeilen durchbohrten, also bereits getöteten Drachen Python steht: Wien. Albertina, Inv. 487 SR 579 R 233, schwarze Kreide, 44,3 × 25,7 cm; vgl. L. Mortari, Francesco Salviati, Rom 1992, S. 277, Nr. 557.

58 Die Kleinbronze im Victoria and Albert Museum (London), Inv. A.99-100, des über ein Seepferd hinwegschreitenden Neptun, der, seitwärts über die rechte Schulter blickend, in Gegendrehung mit dem rechten Arm ausholt, den (fehlenden) Dreizack in Blickrichtung zu stoßen, ähnelt sicher sehr dem oberen Teil der Pose des Danziger Neptuns. Aus der modernen und nicht unbestrittenen Zuschreibung der Londoner Bronze an den in Venedig tätigen Alessandro Vittoria (1525-1608) eine venezianische Herkunft der Figurenerfindung auch der Danziger Figur abzuleiten, wie zuletzt bei M. Karpowicz vorgeschlagen, halte ich jedoch für voreilig. Die Schrittstellung des Londoner Neptun bringt uns vielmehr wieder zu Stoldo Lorenzis Version des Themas für den Brunnen in den Boboli-Gärten. Es wundert mich nicht, die Londoner Bronze alternativ dem in Florenz tätigen Vincenzo Danti (1530-1576) zugeschrieben zu finden. Vgl. M. Karpowicz, Fontanna Neptuna $i$ inspiracje weneckie w sztuce Gdańska, (in:) Ludzie, kontakty, kultura XVI-XVIII w., red. J. Kawecki, J. Tazbir, Warszawa 1997.

59 Vgl. T. Grzybkowska, Między sztuką a polityka. Sala Czerwona Ratusza Gtównego Miasta w Gdańsku, Warszawa 2003, S. 116, Abb.

${ }^{60}$ H. Carl, op. cit., S. 149 (Abb. 2), S. 155. 
Ein derart antikisierende Wahl zu treffen, der die Figurenerfindung ikonographisch und stilistisch zu genügen hatte, setzt voraus, daß diese Kenntnisse und ihre Wertschätzung am Ort vorhanden waren. Daß sich seit 1591 der italienische Humanist und Protestant Giovanni Bernardino Bonifacio Marchese d'Oria (1517-1597) in Danzig aufhielt und 1596 durch Vermächtnis seiner Bücher die erste öffentliche Bibliothek begründete, in der mit Blick auf unser Thema Vergils Aeneis ${ }^{61}$, Ovids Metamorphosen ${ }^{62}$ und wahrscheinlich auch ein Exemplar von Giraldis Mythographie ${ }^{63}$ vertreten waren, könnte sich, bei weiterem Studium, als wichtiges Datum erweisen. Dem Maler des Deckengemäldes aber muß ein konkreter Entwurf vorgelegen haben. Bei dem hochgestreckten Arm, den dieser Neptun aufweist, darf man an ein Beispiel des Niederländers Hubert Gerhard ${ }^{64}$ (s'Hertogenbosch 1550 - München 1620) denken. Er lernte und arbeitete bis 1581 unter Giambologna in Florenz. Von den Fuggern nach Augsburg gerufen, schuf er als ausgewiesener Spezialist für große Bronzen die ersten Monumentalbrunnen des neuen Florentiner Typs im Norden. In seinen Münchner Hauptwerken wiederholte er Giambolognas Hochkontrapost entweder als klassischen Triumphgestus - so bei seinem Perseus (um 1590) im Grottenhof der Residenz und bei dem Erzengel (1588) an der Fassade von St. Michael - oder in Verbindung mit einem Attribut, das als Fußstütze dient, wie zum Beispiel dem Delphin bei dem Neptun 65 (Abb. 14) des Wittelsbacherbrunnens (um 1584-1587). Hoch oben umfaßt er den aufgestellten Dreizack. In der anderen Hand hält er das Muschelhorn, ein Attribut, das sich auch bei dem Danziger Neptun findet. Wenn es indessen um dessen spezifische Bewegungsgestalt geht, wird man ein anderes Beispiel Gerhards konsultieren, den schräg nach unten geführten Lanzenstoß seines Erzengels (1588) an St. Michael in München (Abb. 15), den wir, um zu dem Danziger Resultat zu gelangen, nur in der Bewegungsrichtung des Oberkörpers verändern müßten ${ }^{66}$, während wir die Stellung seiner Beine beibehielten. Sicher

${ }^{61}$ Biblioteka Gdańska Polskiej Akademii Nauk [dalej: BG PAN], Cd. 16663, Cd. 16667; vgl. M. Welti, Die Bibliothek des Giovanni Bernardino Bonifacio, Marchese d'Oria, 15171597. Der Grundstock der Bibliothek Danzig der Polnischen Akademie der Wissenschaften, Bern 1985, S. 376, 413, Nr. 704 u. CBB. 809.

62 BG PAN, Cd. 9560; vgl. M. Welti, op. cit., S. 373, Nr. CBB 702.

${ }^{63}$ BG PAN, Cd. Gb. 4728; vgl. M. Welti, op. cit., Nr. CBB 745.

64 D. Diemer, Hubert Gerhard und Carlo di Cesare del Palagio, Bronzeplastiker der Spätrenaissance, Berlin 2004.

${ }^{65}$ Das Original befindet sich im Bayerischen Nationalmuseum, München.

${ }_{66}$ Michael führte die Lanze mit seiner Rechten von links oben nach rechts unten, der Danziger Neptun dagegen den Dreizack von rechts oben nach links unten, eine Drehbewegung des Rumpfes inbegrifffen. 
kannte Gerhard, vermittelt über den Kupferstich des Beatrizet ${ }^{67}$, Raffaels berühmte Komposition, die Papst Leo X. 1518 an König François I. gesandt hatte und in Frankreich zum Emblem des ritterlichen Michaelsordens geworden war ${ }^{68}$. Gerhard wandelte Raffaels tänzerische Pose ab zu einem Hochkontrapost, bei dem der Engel, ganz nach Florentiner Bewegungsauffassung, das Spielbein bereits abspreizt, um den mit dem Lanzenstoß verbundenen Schwung aufzufangen. Das Muster hierfür können wir in Giambolognas Bronze-Engel69 (Abb. 16) im Skulpturenprogramm (ca. 1581-1587) der Salviati-Kapelle in San Marco (Florenz) erkennen, deren Planungsanfänge Gerhard noch in Florenz bei Giambologna wahrgenommen haben dürfte.

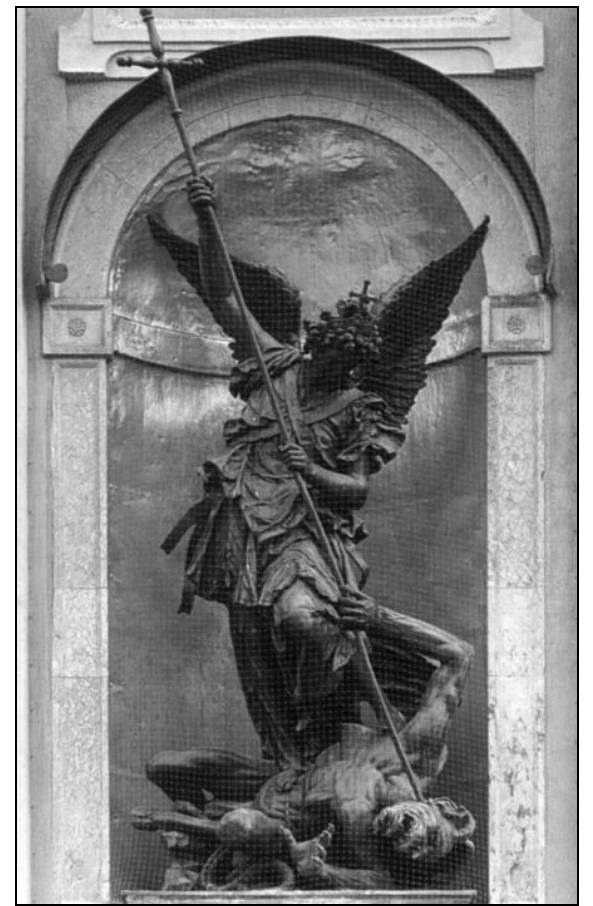

Abb. 15. Hubert Gerhard, Erzengel Michael bezwingt den Dämon, München, St. Michael

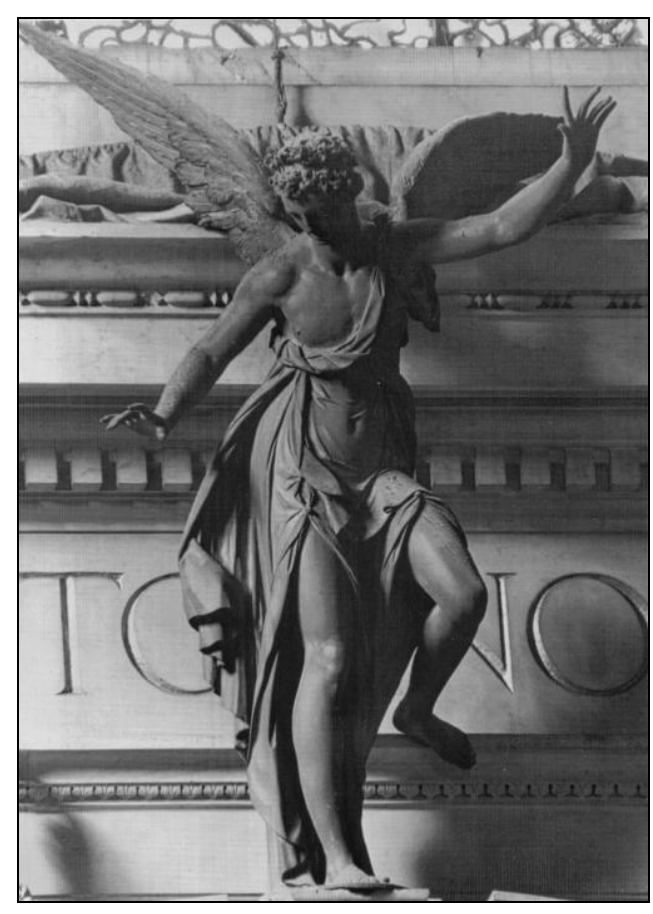

Abb. 16. Giambologna, Engel, Florenz, S. Marco, Cappella Salviati

$67 \mathrm{Zu}$ dem Kupferstich siehe: Raphaël et l'art français, Ausstellung Katalog, Grand Palais, Paris 1983, S. 191 (Nr. 251), S. 191; S. Boorsch, Italian Masters of the Sixteenth Century, New York 1982 (Illustrated Bartsch 29), S. 274, Nr. 30.

${ }_{68} \mathrm{Zu}$ Raphaels Gemälde vgl. Raphaël dans les collections françaises, Ausstellung Katalog, Paris 1983, S. 91-92 (Nr. 9).

69 C. Avery, Giambologna. The complete sculpture, Oxford 1987, S. 200. 
Spätestens 1588 erhielt Hubert Gerhard in Augsburg den Auftrag für den Augustus-Brunnen und leitete damit die Serie bedeutender öffentlicher Bronzebildwerke ein, deren Ruhm die Danziger bewogen haben mag, Unterstützung für ihr Vorhaben dort zu suchen. Zeichnungen des Augsburger Stadtbaumeisters Simon Zwitzel aus dem Jahren 1585 bis 1589 zeugen von der Absicht, den dortigen Fischmarkt-Brunnen unter Verwendung der genannten Neptunfigur - neu zu gestalten ${ }^{70}$. Er wurde 1593, offenbar aufgrund des geringen Abstands zum neuen und repräsentativen Augustusbrunnen, abgebaut und an einem anderen Standort, jedoch wohl in seiner bisherigen Gestalt, wieder errichtet ${ }^{71}$. Gerhards Kunst könnte die Augsburger Ratsherren davon überzeugt haben, Zwitzels biedere Entwürfe nicht umzusetzen. Daß Gerhard selbst sich mit einer Neugestaltung des Augsburger Neptun-Brunnens befaßte, ist nicht dokumentiert. Doch ist seine Alternative vielleicht in Form des Danziger Neptun erhalten. Er scheint sich stilistisch eben in die Reihe der Werke Gerhards der 1580er Jahre zu fügen.

GDAŃSK ON THE MEDITERRANEAN, THE BRONZE SCULPTURE

OF THE FOUNTAIN OF NEPTUNE - ICONOGRAPHY, ARTISTIC INVENTION

AND MEANING

\section{Summary}

The bronze statue of Neptune (around 1615-20) in the Long Market (Długi Targ) in Gdańsk unifies two opposed antique prototypes, a contrapposto figure which shows the god Neptune peacefully resting, and a dynamic figure applying the trident, his weapon, in combat. The new combination represents mimetically the changeable nature of the god's liquid sphere, the sea. In its artistic invention the statue manifests such an exact knowledge and familiarity with the study of antiquity and the artistic methods from Michelangelo Buonarroti until to Giambologna (Jean Boulogne, 15291608), that we have to look for the artist among those trained in sixteenth Century Florence. Among the works of the Dutch Hubert Gerhard (1550-1620), trained until to 1581 in Florence in the circle of Giambologna and later active in Augsburg and Munich as a leading Northern Mannerist artist, the Archangel Vanquishing Lucifer (1588) at the facade of St Michael's Church (Munich) comes most close to the movement expressed in the Gdańsk Neptune.

70 B. Bushart, op. cit., S. 84 ff., Abb. 1-3.

${ }^{71}$ Der Brunnen wurde 1593 auf dem Fischmarkt abgebaut und wohl in weitgehend bisheriger Gestalt in der Weißmalergasse aufgestellt, seit 1745 wieder auf dem Fischmarkt, seit 1888 auf dem Jakobsplatz. 


\section{ABBILDUNGSNACHWEIS}

Abb. 1: Łukasz Golowanow.

Abb. 2: Marcok - it.wikipedia.org.

Abb. 3: Freedman (wie Anm. 12), S. 226.

Abb. 4: Bambach (wie Anm. 11), S. 513.

Abb. 5: Freedman (wie Anm. 12), S. 222.

Abb. 6: Archiv des Verfassers.

Abb. 7: Simon (wie Anm. 31), Bd. 7,2 (Neptun 14).

Abb. 8: Simon (wie Anm. 31), Bd. 7,2 (Poseidon 34b).

Abb. 9: Fuchs (wie Anm. 40), S. 134.

Abb. 10: Simon (wie Anm. 31), Bd. 7,2, Taf. 357.

Abb. 11: Poeschke (wie Anm. 26), S. 204.

Abb. 12: Medri (wie Anm. 48), S. 190.

Abb. 13: Poeschke (wie Anm. 26), fig. 92.

Abb. 14: Rufus46.

Abb. 15: Janiwan.

Abb. 16: Avery (wie Anm. 69), S. 200. 